\title{
Incidence of Hypoglycemia in Patients with Renal Dysfunction Treated for Hyperkalemia with Regular Insulin: A Single Center, Retrospective Cohort Study
}

\section{Dannielle R Brown, PharmD, BCPS ${ }^{1 *}$, Chase C Brown ${ }^{2}$, Tracie A Delay ${ }^{1}$, Genevieve L Hayes ${ }^{1}$ and Ruth C Campbell}

${ }^{1}$ Department of Pharmacy, Medical University of South Carolina, USA

${ }^{2}$ South Carolina College of Pharmacy, Medical University of South Carolina, USA

${ }^{3}$ Department of Nephrology, Medical University of South Carolina, USA

*Corresponding author: Dannielle R Brown, Department of Pharmacy, Medical University of South Carolina, 150 Ashley Avenue, Charleston, SC 29425, USA, Tel: (757)-409-5474, Fax: (843)-792-3759

\begin{abstract}
Objective: To identify the incidence of and risk factors for hypoglycemia in patients with chronic kidney disease (CKD) stage III-V or end stage renal disease (ESRD) treated with intravenous (IV) insulin for hyperkalemia.

Design: Single-center, retrospective, cohort study.

Setting: Large academic tertiary care medical center.

Patients: Adults with a diagnosis of CKD stage III-V or ESRD who had a serum potassium $\geq 5 \mathrm{mmol} / \mathrm{L}$ and were treated with regular insulin for hyperkalemia between October 1, 2015 and August 16, 2016.

Measurements and main results: The primary outcome was the incidence of hypoglycemia during the hospitalization after the administration of insulin for hyperkalemia. Key secondary outcomes included incidence of hypoglycemia within 24 hours of insulin administration and identification of risk factors associated with hypoglycemia. Data collected included patient age, sex, race, history of diabetes, stage of CKD, weight, body mass index (BMI), baseline blood glucose, inciting serum potassium concentration, units of regular insulin administered, and grams of dextrose given with insulin. A total of 235 hospitalizations were included in the final analysis. Sixty-six patients $(28.1 \%)$ experienced a hypoglycemic event during their hospital encounter. Fifty-three patients $(22.6 \%)$ had hypoglycemia within 24 hours of insulin administration. The average time to onset of hypoglycemia was 21.2 hours in all patients who experienced hypoglycemia and 6.4 hours in the subset of patients who experienced hypoglycemia within 24 hours of insulin administration. Inciting potassium was significantly higher and
\end{abstract}

baseline blood glucose was significantly lower in the group that experienced hypoglycemia $(P=0.01, P=0.02$, respectively).

Conclusion: Hypoglycemia following treatment for hyperkalemia with IV regular insulin was common in this patient population. Consideration should be given to the development of a comprehensive order set for insulin use in hyperkalemia to include protocol driven glucose monitoring with dextrose administration tailored to baseline glucose levels.

\section{Keywords}

Hyperkalemia, Hypoglycemia, Insulin, Chronic kidney disease, End stage renal disease

Patients with decreased renal function are at an increased risk of experiencing hyperkalemia [1]. As glomerular filtration rate (GFR) decreases, secretion, filtration, and elimination of potassium also declines [2]. Additionally, chronic kidney disease (CKD) patients are frequently on medications that can potentiate hyperkalemia such as renin-angiotensin-aldosterone system (RAAS)-blocking agents [3]. Mild hyperkalemia is often asymptomatic; however, severe potassium elevations can lead to cardiotoxicity and fatal dysrhythmias $[4,5]$. Acute management of symptomatic hyperkalemia includes stabilization of the cardiac membrane with intravenous (IV) calcium gluconate and rapid redistribution of potassium to the intracellular space with inhaled or

\footnotetext{
Citation: Brown DR, Brown CC, Delay TA, Hayes GL, Campbell RC (2019) Incidence of Hypoglycemia in Patients with Renal Dysfunction Treated for Hyperkalemia with Regular Insulin: A Single Center, Retrospective Cohort Study. Int Arch Clin Pharmacol 5:018. doi.org/10.23937/2572-3987.1510018 Accepted: June 01, 2019: Published: June 03, 2019

Copyright: (c) 2019 Brown DR, et al. This is an open-access article distributed under the terms of the Creative Commons Attribution License, which permits unrestricted use, distribution, and reproduction in any medium, provided the original author and source are credited.
} 
IV beta agonists (eg, albuterol) and/or IV regular insulin $[4,5]$. The American Heart Association (AHA) guidelines for cardiopulmonary resuscitation and the European Resuscitation Council Guidelines both recommend 10 units of regular insulin for the management of severe hyperkalemia, administered with 25 grams of dextrose to prevent hypoglycemia $[4,5]$. Both sets of guidelines recommend blood glucose monitoring after insulin administration but do not specify the frequency or duration of monitoring $[4,5]$.

While insulin is an effective treatment for acute hyperkalemia, it is also a high-risk medication due to the potential for hypoglycemia [6]. Insulin undergoes renal elimination, resulting in increased hypoglycemic risk in patients with CKD due to prolonged effects [7]. Previous retrospective studies have shown that nearly $20 \%$ of patients with CKD and/or end stage renal disease (ESRD) treated with insulin and dextrose for hyperkalemia experience hypoglycemic events [8-10]. In one study, hypoglycemia occurred as long as 7.5 hours after insulin administration [10].

At MUSC Health, there have been numerous anecdotal reports of persistent hypoglycemia in patients with renal dysfunction treated with insulin and dextrose for hyperkalemia. MUSC Health does not currently have standardized protocols for treating hyperkalemia or monitoring blood glucose values after IV insulin administration. This study was conducted to assess the incidence of hypoglycemia at MUSC Health, with the ultimate goal of establishing a protocol to safely and effectively treat hyperkalemia. We also sought to identify additional risk factors for hypoglycemia in this patient population.

\section{Methods}

\section{Study design, setting, and patients}

This retrospective study was conducted in patients 18 years of age or older with a diagnosis of CKD stage III, IV, V, or ESRD who were treated with regular insulin for a serum potassium $\geq 5 \mathrm{mmol} / \mathrm{L}$ between October 1 , 2015 and August 16, 2016 at a large academic tertiary care medical center. Patients were identified by ICD-10 codes. Patients were excluded from the study if they did not have a documented blood glucose reading within 24 hours before insulin administration and within 24 hours after insulin administration. Patients with baseline blood glucose values $\leq 70 \mathrm{mg} / \mathrm{dL}$ were also excluded. Hypoglycemia was defined as a blood glucose value $<70 \mathrm{mg} / \mathrm{dL}$, and severe hypoglycemia was defined as a blood glucose value $<50 \mathrm{mg} / \mathrm{dL}$.

\section{Data collection}

Data were collected via electronic medical record chart review. Data collected included patient age (years), sex, race, history of diabetes, stage of CKD, kidney transplant status, weight $(\mathrm{kg})$, body mass index (BMI) $(\mathrm{kg} /$ $\mathrm{m}^{2}$ ), baseline blood glucose $(\mathrm{mg} / \mathrm{dL})$, inciting potassi- um ( $\mathrm{mmol} / \mathrm{L})$, units of regular insulin administered, and grams of dextrose given with insulin. Patients' post-insulin glucose values were collected for the remainder of patient's hospitalization.

\section{Outcomes and statistical analysis}

The primary outcome was the incidence of hypoglycemia during the patient's hospitalization after the administration of insulin for the treatment of hyperkalemia. Secondary outcomes included the incidence of hypoglycemia within 24 hours of insulin administration and identification of risk factors associated with hypoglycemia. Categorical data was assessed via Chi-squared or Fischer's exact test, as appropriate, and continuous variables were analyzed via student t-test.

\section{Results}

A total of 334 encounters where intravenous regular insulin was administered to treat hyperkalemia in patients with CKD Stages III-V or ESRD were identified. After exclusion of 99 encounters (Table 1), 235 hospitalizations were examined for the incidence of hypoglycemia and included in the final analysis. These hospitali-

Table 1: Reasons for Exclusion $(n=99)$.

\begin{tabular}{|l|l|}
\hline Reason & $\mathbf{n , ~ ( \% )}$ \\
\hline Age $<18$ years & $2(2.0)$ \\
\hline Baseline glucose $<70 \mathrm{mg} / \mathrm{dL}$ & $7(7.1)$ \\
\hline Potassium $<5 \mathrm{mmol} / \mathrm{L}$ & $7(7.1)$ \\
\hline $\begin{array}{l}\text { No glucose reading }<24 \text { hours after insulin } \\
\text { administration }\end{array}$ & $9(9.1)$ \\
\hline $\begin{array}{l}\text { No glucose reading }<24 \text { hours before insulin } \\
\text { administration }\end{array}$ & $15(15.2)$ \\
\hline No insulin administration & $26(26.2)$ \\
\hline No diagnosis of CKD & $33(33.3)$ \\
\hline
\end{tabular}

Table 2: Dosing of Insulin and Concomitant Dextrose.

\begin{tabular}{|l|l|}
\hline Agent and Dose & $\mathbf{n , ~ ( \% )}$ \\
\hline Insulin & \\
\hline 2.5 units & $1(0.4)$ \\
\hline 4 units & $1(0.4)$ \\
\hline 5 units & $33(14.0)$ \\
\hline 6 units & $3(1.3)$ \\
\hline 7 units & $1(0.4)$ \\
\hline 7.5 units & $1(0.4)$ \\
\hline 8 units & $2(0.9)$ \\
\hline 10 units & $191(81.3)$ \\
\hline 20 units & $2(0.9)$ \\
\hline Dextrose (given with insulin) & \\
\hline None & $23(9.8)$ \\
\hline 12.5 grams & $2(0.8)$ \\
\hline 15 grams & $1(0.4)$ \\
\hline 25 grams & $194(82.6)$ \\
\hline 50 grams & $15(6.4)$ \\
\hline & \\
\hline
\end{tabular}


zations included a total of 206 patients. The number of patients with multiple treatment episodes analyzed are as follows: 14 patients with 2 hospitalizations each, 6 patients with 3 hospitalizations each, and 1 patient with 4 hospitalizations. The doses of insulin and concomitant dextrose use are listed in Table 2. Insulin doses ranged from 2.5 units to 20 units. The majority of patients received 10 units of insulin (81.3\%) and 25 grams of dextrose $(82.6 \%)$, though there was a small proportion where dextrose was not administered concomitantly with insulin (9.8\%).
Of the analyzed encounters, $28.1 \%(n=66 / 235)$ experienced a hypoglycemic event during the encounter, with nadir blood glucose readings ranging from $20 \mathrm{mg} /$ $\mathrm{dL}$ to $69 \mathrm{mg} / \mathrm{dL}$. Of those, $80.3 \%$ (53/66) had the episode of hypoglycemia within 24 hours of insulin administration. The incidence of severe hypoglycemia among the 235 analyzed encounters was $9.8 \%(n=23 / 235)$. These 23 episodes of severe hypoglycemia occurred in a total of 20 patients. Figure 1, demonstrates the course of blood glucose readings in 17 of the severe hypoglycemia episodes ( 3 not included due to receiving multi-

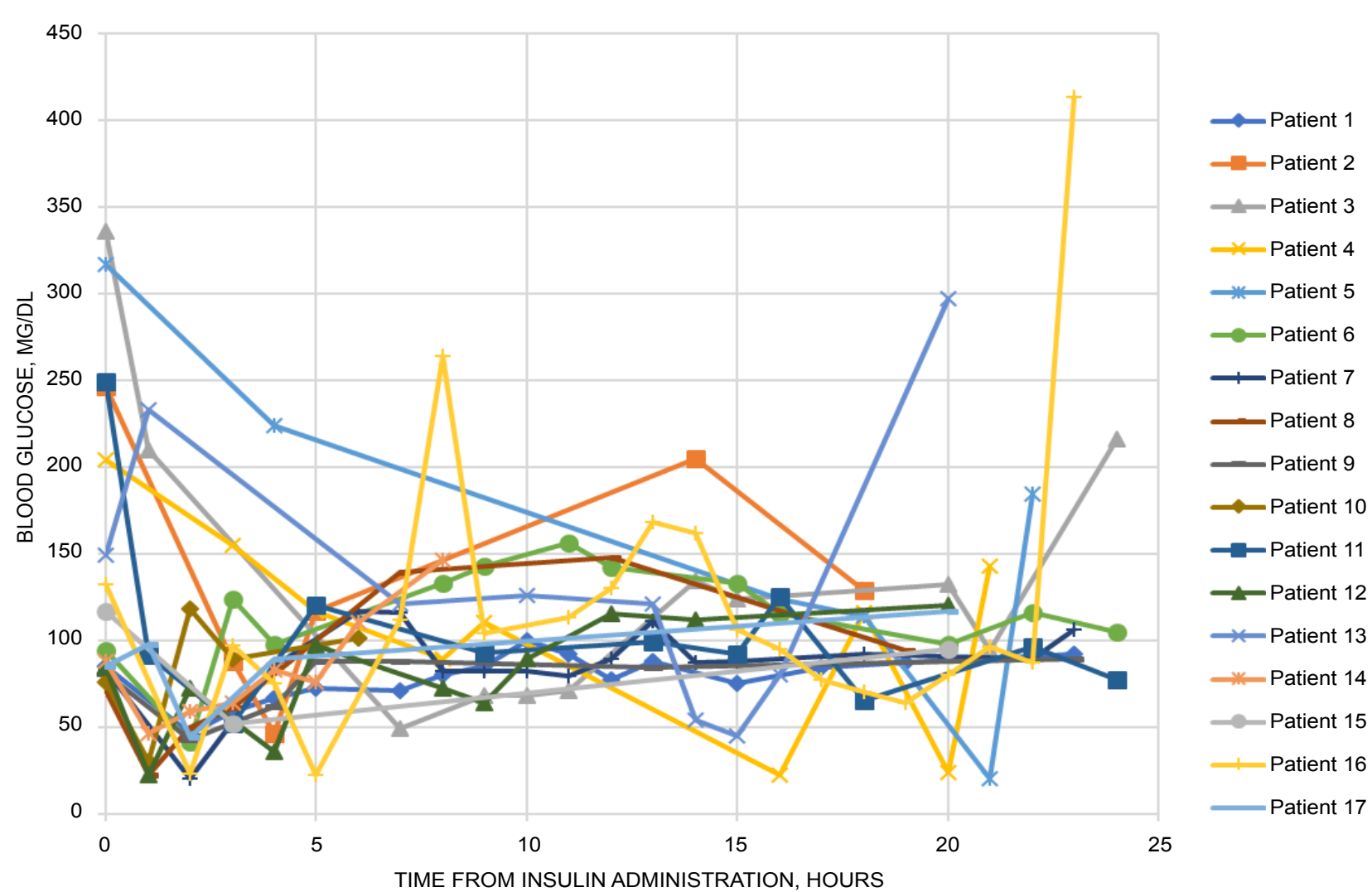

Figure 1: Severe hypoglycemia within 24 hours of insulin administration. The course of blood glucose readings in 17 of 23 encounters with an episode of severe hypoglycemia (blood glucose $<50 \mathrm{mg} / \mathrm{dL}$ after insulin administration for the treatment of hyperkalemia). The incidence of severe hypoglycemia was $9.8 \%$. Only 17 of the severe hypoglycemia episodes were analyzed (3 not included due to receiving multiple doses of insulin, 3 not included because the first low blood glucose reading was $>24$ hours after insulin administration). These 17 episodes occurred in a total of 13 patients (one patient with 3 separate episodes, 2 patients with 2 separate episodes each, and 10 patients with 1 episode each during the study period). Of the 17 analyzed episodes, the average baseline blood glucose value was $137 \mathrm{mg} / \mathrm{dL}$. Dextrose was administered concomitantly with insulin at a dose of 50 grams for 1 episode, 25 grams for 15 episodes, and no dextrose was administered in one episode.

Table 3: Comparison of Patient Characteristics between Hypoglycemia and No Hypoglycemia Cohorts.

\begin{tabular}{|l|l|l|l|}
\hline & No Hypoglycemia $(\mathbf{n}=169)$ & Hypoglycemia $(\mathbf{n}=66)$ & P value \\
\hline Age, mean (SD), y & $55.8(15.9)$ & $59.6(16.3)$ & 0.11 \\
\hline Male, $\mathrm{n}(\%)$ & $95(56.2)$ & $42(63.6)$ & 0.42 \\
\hline African American, $\mathrm{n}(\%)$ & $112(66.3)$ & $53(80.3)$ & 0.06 \\
\hline History of Diabetes, $\mathrm{n}(\%)$ & $91(53.8)$ & $34(51.5)$ & 0.93 \\
\hline Stage V CKD, $\mathrm{n}(\%)$ & $96(56.8)$ & $41(62.1)$ & 0.55 \\
\hline Weight, mean (SD), kg & $83.7(27.8)$ & $79.8(24.1)$ & 0.32 \\
\hline BMl, mean (SD), kg/m² & $28.5(8.4)$ & $27.7(6.4)$ & 0.52 \\
\hline Inciting Potassium Level, mean (SD), mmol/L & $6.4(0.7)$ & $6.6(0.8)$ & 0.01 \\
\hline Baseline Blood Glucose, mean (SD), mg/dL & $187.3(170.5)$ & $136.6(80.2)$ & 0.02 \\
\hline
\end{tabular}


ple doses of insulin, 3 not included because the first low blood glucose reading was $>24$ hours after insulin administration). The average time to onset of hypoglycemia was 21.2 hours in all patients who experienced hypoglycemia and 6.4 hours in the subset of patients who experienced hypoglycemia within 24 hours of insulin administration. Over half of the patients who experienced hypoglycemia required treatment to correct blood glucose levels $(53 \%, n=35 / 66)$.

Baseline characteristics between patients who did not experience an episode of hypoglycemia during the encounter and those that did were relatively well matched (Table 3). Most patients were classified as CKD Stage V (Figure 2). Notably, $12 \%$ of patients were either pre- or post-kidney transplant. There was no significant difference in mean age, percentage of male gender, percentage of African American, history of diabetes, Stage V CKD, mean weight, or mean BMI (Table 3). However, there was a significant difference in inciting potassium concentration between the two groups (mean $6.4 \mathrm{mmol} / \mathrm{L}$ in the non-hypoglycemic group versus mean $6.6 \mathrm{mmol} / \mathrm{L}$ in the hypoglycemic group, $\mathrm{P}=$ 0.01 ). Additionally, there was a significant difference in the baseline blood glucose values (mean $187.3 \mathrm{mg} / \mathrm{dL}$ in the group who did not experience hypoglycemia versus mean $136.6 \mathrm{mg} / \mathrm{dL}$ in the group who did experience hypoglycemia, $P=0.02$ ). Most patients who experienced hypoglycemia received 25 grams of dextrose with the insulin dose $(83.3 \%, n=56 / 66)$ and only one patient received no dextrose with the insulin dose.

\section{Discussion}

Treatment of patients with hyperkalemia, a common complication in CKD Stages III-V or ESRD, with regular insulin is often associated with the development of hypoglycemia. Published guidelines recommend a dose

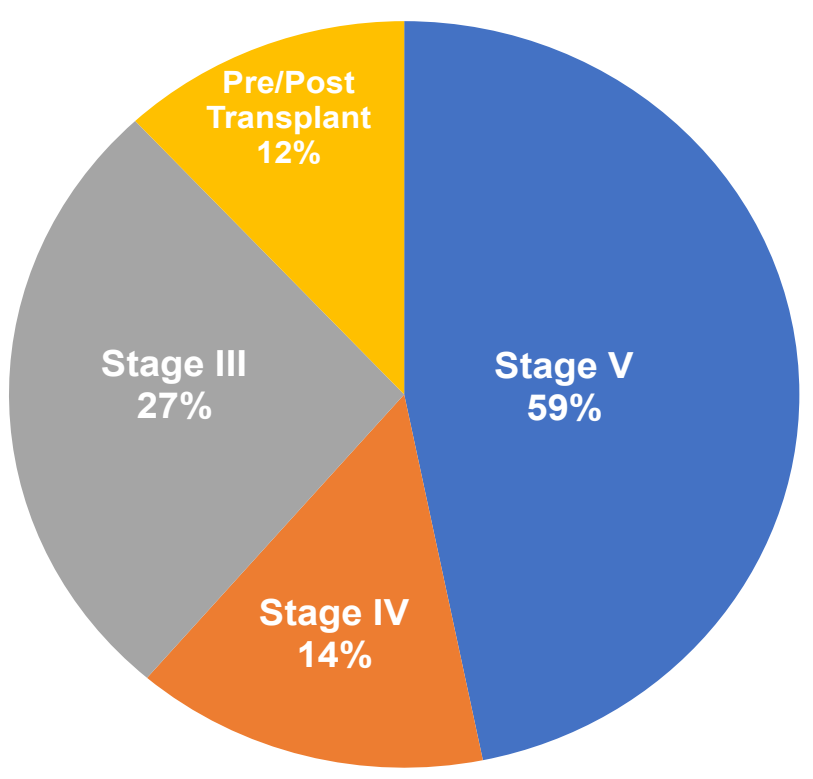

Figure 2: Stages of CKD. A majority of patients included in this study had Stage V CKD based on ICD-10 codes. of 10 units of regular insulin intravenously for the treatment of hyperkalemia, given with 25 grams of dextrose to prevent hypoglycemia $[4,5]$. However, several small studies have identified varying rates of hypoglycemia after treatment with insulin for hyperkalemia even with concomitant dextrose administration [8-10]. Pierce, et al. specifically studied the rates of hypoglycemia in patients with low estimated glomerular filtration rates treated with either low-dose (5 units) or high-dose (10 units) insulin [10]. The study demonstrated an overall $18.1 \%$ rate of hypoglycemia and no difference in this rate between high or low doses of insulin in patients with low eGFR ( $16.7 \%$ in the high dose group versus $19.7 \%$ in the low-dose group, $P=0.79$ ) [10]. In response to a number of hypoglycemic events at MUSC Health, this study was designed to investigate potential risk factors for the development of hypoglycemia after treatment with insulin in CKD Stage III-V and ESRD patients and to determine if a specific protocol is needed in this population.

Results demonstrated a $28.1 \%$ overall rate of hypoglycemia in this study, and the rate of severe hypoglycemia was $9.8 \%$, despite the large majority having received dextrose concomitantly with insulin $(95.5 \%, \mathrm{n}$ $=63 / 66)$. Most received 25 grams of dextrose $(84.8 \%$, $\mathrm{n}=56 / 66$ ). Initial blood glucose concentrations in those who developed hypoglycemia ranged from $71 \mathrm{mg} /$ $\mathrm{dL}$ to $454 \mathrm{mg} / \mathrm{dL}$, with a mean baseline blood glucose significantly lower than the mean baseline blood glucose in the group who did not experience hypoglycemia. Though not statistically significant when compared to patients who did not experience a hypoglycemic effect, the patients who developed hypoglycemia tended to be older and weigh less, with a higher percentage that were African Americans with CKD Stage V; fewer patients had history of diabetes. There was wide variability in the timing and frequency of blood glucose monitoring before and after insulin administration. Nil per os (NPO) or nothing by mouth status was not examined in this study and could potentiate hypoglycemia in this patient population.

To improve hypoglycemia rates, consideration should be given to the development of a computerized order set for the use of intravenous regular insulin for the treatment of hyperkalemia that would include a standard schedule for glucose monitoring and options for dextrose dosing that depend on baseline blood glucose values. In addition, more stringent glucose monitoring for at least the first 7 hours after insulin administration given the average time to onset of 6.4 hours in the subset of patients who experienced hypoglycemia within the first 24 hours is warranted.

There were several limitations within this study. First, the study was designed as a retrospective chart review at a single academic medical center for a limited duration of time with a small number of patients 
in each group, which limits the ability to detect a difference between the groups. As a retrospective chart review, the study depended on complete and accurate documentation within the electronic medical record and underreporting of any values could have affected study results. Secondly, given the lack of protocoled treatment at the institution, there was a wide variability in the dosing of insulin, dosing of dextrose, and timing of blood glucose measurements. Such variability made it difficult to determine when hypoglycemia truly occurred and potentially affected the determination of incidence. We reported the rate of hypoglycemia throughout the patient's hospitalization, likely over-estimating hypoglycemia directly resulting from hyperkalemia treatment. In addition, the change in potassium concentrations was not studied between the 2 groups, other hyperkalemia treatments were not noted, and confounding factors such as additional insulin and dextrose administration were not recorded. Third, patients without glucose values before and after insulin administration for hyperkalemia were excluded. This may be a source of bias that potentially overestimates or underestimates the risk of hypoglycemia following insulin administration. Finally, the study did not assess the duration of hypoglycemic episodes due, in part, to the fact that the timing of blood glucose measurements was not standardized.

\section{Conclusion}

The complication of hypoglycemia in CKD Stages III-V or ESRD patients treated with regular insulin for hyperkalemia was a common occurrence in this study. Additionally, several of the patients studied developed hypoglycemia on multiple encounters after treatment with regular insulin suggesting that they have risk factors leading to these recurrent episodes. Though there was only a significant difference in the inciting potassium level and baseline blood glucose values between the group who experienced hypoglycemia and those who did not, the results of the study are still telling. Current practice at MUSC Health has an opportunity to incorporate protocols for the standardization in both insulin/dextrose dosing administration and in follow-up glucose monitoring. Development of a protocol could help to minimize hypoglycemia risk. Given patient variability and the wide range of baseline blood glucose values seen in this patient population, focus on sufficient dextrose dosing dependent on baseline blood glu- cose value with more standardized glucose monitoring before and after insulin administration is warranted.

\section{Conflicting Interests}

The authors have no real or potential conflicts of interests to declare. The authors received no financial support for the research or publication of the poster or article.

\section{Funding}

No source of funding was available for this study.

\section{Presentation}

Results were presented as a poster at American Society of Health-System Pharmacists Midyear Clinical Meeting, Las Vegas, NV, December 7, 2016.

\section{References}

1. Einhorn LM, Zhan M, Hsu VD, Walker LD, Moen MF, et al. (2009) The frequency of hyperkalemia and its significance in chronic kidney disease. Arch Intern Med 169: 1156-1162.

2. Allon M (1995) Hyperkalemia in end-stage renal disease: Mechanisms and management. J Am Soc Nephrol 6: 11341142.

3. Nakhoul GN, Huang $H$, Arrigain S, Jolly SE, Schold JD, et al. (2015) Serum potassium, end-stage renal disease and mortality in chronic kidney disease. Am J Nephrol 41: 456463.

4. (2005) 2005 American Heart Association guidelines for cardiopulmonary resuscitation and emergency cardiovascular care. Circulation 112: 1-5.

5. Soar J, Perkins GD, Abbas G, Alfonzo A, Barelli A, et al. (2010) European resuscitation council guidelines for resuscitation 2010 section 8. cardiac arrest in special circumstances: Electrolyte abnormalities, poisoning, drowning, accidental hypothermia, hyperthermia, asthma, anaphylaxis, cardiac surgery, trauma, pregnancy, electrocution. Resuscitation 81 : 1400-1433.

6. (2014) ISMP's List of High-Alert Medications.

7. (2015) Humulin R [prescribing information]. Eli Lilly and Company, Indianapolis, USA.

8. Apel J, Reutrakul S, Baldwin D (2014) Hypoglycemia in the treatment of hyperkalemia with insulin in patients with endstage renal disease. Clin Kidney J 7: 248-250.

9. Schafers S, Naunheim R, Vijayan A, Tobin G (2012) Incidence of hypoglycemia following insulin-based acute stabilization of hyperkalemia treatment. J Hosp Med 7: 239-242.

10. Pierce DA, Russell G, Pirkle JL (2015) Incidence of hypoglycemia in patients with low eGFR treated with insulin and dextrose for hyperkalemia. Ann Pharmacother 49: $1322-1326$.

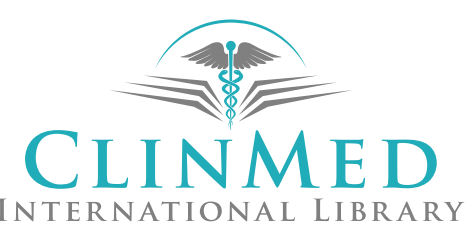

